\title{
The forkhead transcription factor Foxo1 (Fkhr) confers insulin sensitivity onto glucose-6-phosphatase expression
}

\author{
Jun Nakae, ${ }^{1}$ Tadahiro Kitamura, ${ }^{1}$ David L. Silver, ${ }^{2}$ and Domenico Accili ${ }^{1}$ \\ ${ }^{1}$ Naomi Berrie Diabetes Center, and \\ ${ }^{2}$ Division of Molecular Medicine, Department of Medicine, College of Physicians and Surgeons of Columbia University, \\ New York, New York, USA
}

Address correspondence to: Domenico Accili, Berrie Research Pavilion, 1150 Street, Nicholas Avenue, Room 238, New York, New York 10032, USA. Phone: (212) 304-7391; Fax: (212) 304-7390; E-mail: da230@columbia.edu.

Received for publication April 2, 2001, and accepted in revised form September 4, 2001.

\begin{abstract}
Type 2 diabetes is characterized by the inability of insulin to suppress glucose production in the liver and kidney. Insulin inhibits glucose production by indirect and direct mechanisms. The latter result in transcriptional suppression of key gluconeogenetic and glycogenolytic enzymes, phosphoenolpyruvate carboxykinase (Pepck) and glucose-6-phosphatase (G6p). The transcription factors required for this effect are incompletely characterized. We report that in glucogenetic kidney epithelial cells, Pepck and G6p expression are induced by dexamethasone (dex) and cAMP, but fail to be inhibited by insulin. The inability to respond to insulin is associated with reduced expression of the forkhead transcription factor Foxo1, a substrate of the Akt kinase that is inhibited by insulin through phosphorylation. Transduction of kidney cells with recombinant adenovirus encoding Foxo1 results in insulin inhibition of dex/cAMP-induced G6p expression. Moreover, expression of dominant negative Foxo1 mutant results in partial inhibition of dex/cAMP-induced G6p and Pepck expression in primary cultures of mouse hepatocyes and kidney LLC-PK1-FBPase ${ }^{+}$cells. These findings are consistent with the possibility that Foxo1 is involved in insulin regulation of glucose production by mediating the ability of insulin to decrease the glucocorticoid/cAMP response of G6p.
\end{abstract}

J. Clin. Invest. 108:1359-1367 (2001). DOI:10.1172/JCI200112876.

\section{Introduction}

Type 2 diabetes mellitus results from impaired insulin action and inadequate insulin secretion (1). A key abnormality in the pathogenesis of diabetes is insulin's failure to restrain endogenous glucose production, resulting in increased blood glucose levels (2). Evidence from clamped dogs (2) and genetically engineered mice indicates that insulin action on glucose production includes indirect and direct effects $(3,4)$. For example, hepatocytes lacking insulin receptors lack the ability to suppress glucose output in response to insulin and display increased Pepck and G6p levels $(5,6)$. The primary sites of glucose production are the liver and kidney $(2$, 7). In vivo studies indicate that during prolonged fasting or diabetes, renal glucose production can account for up to $25 \%$ of total endogenous glucose production (8-13), although other studies suggest a much lower contribution $(14,15)$. Renal glucose production, like hepatic glucose production, is suppressed by insulin in vivo (10, 16-18). Insulin's ability to reduce glucose production is preserved in cultured hepatocytes, but not in cultured kidney epithelial cells, the site of renal glucose production. The latter display hormonal $(19,20)$ and $\mathrm{pH}$-regulated gluconeogenesis (20-29), but have never been shown to be sensitive to insulin inhibition.

Insulin controls glucose production by inhibiting expression of two rate-limiting enzymes in gluconeogenesis and glycogenolysis, Pepck and G6p $(30,31)$. The signaling pathways required for these effects are incom- pletely understood, but are thought to require activation of the lipid kinase phosphatidylinositol 3 (PI 3-kinase) (32-35). Among the PI-dependent kinases, Akt has been implicated as a mediator of insulin's inhibition of a reporter gene driven by a Pepck promoter spanning the putative insulin response sequence (IRS) (36). However, the use of different dominant negative Akt mutants has yielded controversial results $(34,37)$. The forkhead transcription factor Foxo1 (previously known as Fkhr) (38) is phosphorylated in an insulin-responsive manner by $\mathrm{PIP}_{3}$-dependent kinases, such as Akt and Sgk (39-50). Phosphorylation leads to nuclear exclusion and inhibition of Foxo1-dependent transcription (39-42, 44-55). Studies in hepatoma cells suggest that Foxo1 and its closely related isoform Foxo3 possess the ability to regulate transcription of reporter genes containing insulin response elements from the G6p and Pepck promoters in an insulin-dependent manner $(56,57)$. However, it is unclear whether the endogenous genes can be regulated in a similar manner and whether Foxo proteins are the physiologic mediators of insulin action on Pepck and G6p. To address this question, we characterized the hormonal regulation of Pepck and G6p expression in LLC-PK1-FBPase ${ }^{+}$kidney epithelial cells. In this study we show that Pepck and G6p in these cells are refractory to insulin inhibition. This refractoriness is associated with low levels of Foxo1 expression. Expression of Foxo1 by adenovirus-mediated gene transfer confers insulin inhibition onto the dex/cAMP cock- 
tail-induced (see Methods) rise in G6p. Moreover, a dominant negative Foxo1 lacking the transactivation domain partially inhibits dex/cAMP cocktail-induced G6p and Pepck expression both in LLC-PK1-FBPase ${ }^{+}$ cells and in primary cultures of mouse hepatocytes. We suggest that Foxo1 plays an important role in insulin control of G6p expression and that dominant negative Foxo1 mutants provide a useful reagent to inhibit glucogenesis in experimental systems.

\section{Methods}

Reagents. LLC-PK1-FBPase ${ }^{+}$cells are a glucogenetic substrain of LLC-PK1 cells that express fructose-bisphosphatase $(58,59)$. Although they are different from the parental LLC cell line, for brevity we refer to them as LLC cells. The following cDNA probes were obtained: G6p, Pepck, FOXA2 (Hnf-3ß), HNF-1 $\alpha$, and serum- and glucocorticoid-induced kinase 2 (SGK 2). Probes for Foxo1, Foxo3, and Foxo4 were described previously (45). Gapdh and $\beta$-actin were prepared by RT-PCR using Gene Amp RNA PCR kit (Applied Biosystems, Foster City, California, USA) according to the manufacturer's protocol.

Cell culture and $A b^{\prime}$ s. LLC cells were cultured at $37^{\circ} \mathrm{C}$ in DMEM with $5.5 \mathrm{mM}$ D-glucose (Life Technologies Inc., Rockville, Maryland, USA), $2 \mathrm{mM}$ glutamine, and 44 $\mathrm{mM} \mathrm{NaHCO}_{3}$ supplemented with $10 \%$ FBS (Mediatech Inc., Herndon, Virginia, USA), $100 \mathrm{U} / \mathrm{ml}$ penicillin, and $100 \mu \mathrm{g} / \mathrm{ml}$ streptomycin. For induction of G6p and Pepck mRNA, cells were incubated with DMEM supplemented with $0.1 \%$ BSA, $0.5 \mathrm{mM}$ 8-bromoadenosine $3^{\prime}: 5^{\prime}$-cyclic monophosphate (8-Br-cAMP) (Sigma Chemical Co., St. Louis, Missouri, USA), $0.5 \mathrm{mM}$ 3-isobutyl1-methylxanthine (IBMX) (Sigma Chemical Co.), and 1 $\mu \mathrm{M}$ dexamethasone (Sigma Chemical Co.) for 0-24 hours. This cocktail is referred to throughout as dex/cAMP. Anti-c-Myc mAb 9E10 and anti-hemagglutinin (anti-HA) mAB 12CA5 were obtained from Santa Cruz Biotechnology Inc. (Santa Cruz, California, USA) and Boehringer Mannheim GmbH (Mannheim, Germany),respectively. Rabbit polyclonal antisera against FOXO1-S ${ }^{256}$, FOXO4-T28, and FOXO1-S 318 phosphopeptides were from Cell Signaling Technologies (Beverly, Massachusetts, USA). Ab's were used for Western blot analysis at 1:1,000 dilution.

Hepatocyte isolation. Hepatocytes were isolated according to the method of Honkakoski (60), with the following modifications: Complete protease inhibitor (Boehringer Mannheim $\mathrm{GmbH}$ ) was added to digestion buffer according to the manufacturer's specifications (61). Hormonal treatment was carried out in DMEM supplemented with dex/cAMP for 4 hours.

Construction of adenoviral vectors. To facilitate identification of Foxo1 expressed with adenoviral vectors, a HA epitope tag was cloned at the $5^{\prime}$ of the murine cDNAs encoding wild-type (WT), T24A/S253D/S316A (ADA), and $\triangle 256$ mutant Foxo1. To this end, PCR reactions were performed using the respective Foxo $1 \mathrm{cDNA}$ as templates. The same upstream (sense) primer was used in all three constructs: $5^{\prime}$-ACT GGT ACC GCC ATG TAC CCA TAC GAT GTT CCG GAT TAC GCT GCC GAG GCG CCC CAG GTG GTG G-3'. Two different antisense primers were used: $5^{\prime}$-TTG CCC CAC GCG TTG CGG CGC GAC GAG C-3' (to construct WT and ADA Foxo1) and $5^{\prime}$-AAT TCT AGA GTC CAT GGA CGC AGC TCT TCT CCG$3^{\prime}$ (to construct the $\Delta 256$ mutant) (47). The resulting PCR fragments were digested with KpnI and MluI and subcloned into KpnI/MluI-treated pCMV5/c-Myc WT and ADA Foxo1. For the construction of HA-tagged $\Delta 256$ Foxo1, after digestion with KpnI and XbaI, the PCR fragment was subcloned into KpnI- and XbaItreated pCMV5/c-Myc. DNA encoding the HA-tagged WT and mutant Foxo1 was subcloned into pAxCAwt, and adenovirus vectors containing these cDNAs were generated by transfecting HEK 293 cells with the corresponding pAxCAwt plasmid, together with a DNAterminal protein complex (62). Experiments were carried out 24 and 48 hours after infection in hepatocytes and LLC cells, respectively.

Construction of c-Myc-tagged Foxo3 expression vector. A full-length mouse cDNA clone encoding Foxo3 was assembled by subcloning two separate fragments into

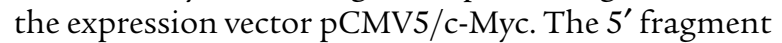
was obtained by PCR using a cloned Foxo 3 cDNA as template. The primers used are: upstream, $5^{\prime}$-GGG GAA TTC ATG GCA GAG GCA CCA GCC TCC-3', nucleotide (nt) 326-343, and downstream, 5'-GTC GCC CTT ATC CTT GAA GTA-3' (nt 922-902). The PCR product was digested with EcoRI and BglII and cloned into EcoRI/BglII-digested pCMV5/c-Myc. The $3^{\prime}$ fragment, encompassing nt 865-2886 of the Foxo3 sequence, was obtained by digestion of a full-length cDNA clone with BglII and HindIII and ligated into the same sites of the pCMV5/c-Myc. The expression vector was fully sequenced before transfection to confirm that no mutations had been introduced during the cloning procedure.

$m R N A$ isolation and Northern blot analysis. Cells were incubated in serum-free medium supplemented with dex/cAMP for 16 hours. Thereafter, insulin was added to the medium for up to 6 hours at a final concentration of $100 \mathrm{nM}$. Cells were harvested by trypsinization, and mRNA was isolated using MicroFast Track 2.0 kit (Invitrogen Corp., San Diego, California, USA). mRNA was size-fractionated on denaturing formaldehyde/agarose gels and transferred to a nylon membrane for Northern hybridization according to standard techniques.

Immunodetection of insulin and IGF-1 receptors. Cells were harvested from a $10-\mathrm{cm}$ culture dish and solubilized in Triton X-100. Detergent cell extracts were immunoprecipitated with anti-insulin receptor (anti-IR) Ab Ab-3 (Calbiochem-Novabiochem Corp., San Diego, California, USA) or anti-IGF-1R Ab C-20 (Transduction Laboratories, Lexington, Kentucky, USA) and blotted with anti-IR Ab C-19 or anti-IGF-1R Ab C-20 (Transduction Laboratories) as described previously (47). Antisera were used at a dilution of 1:1,000. 
a

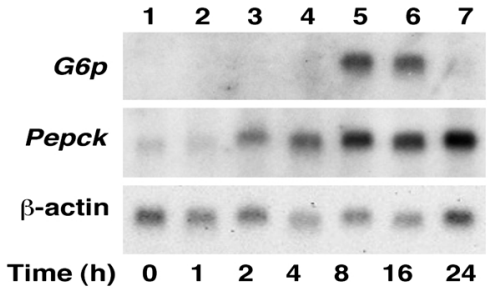

b

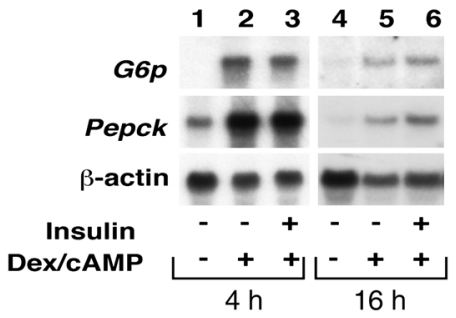

C

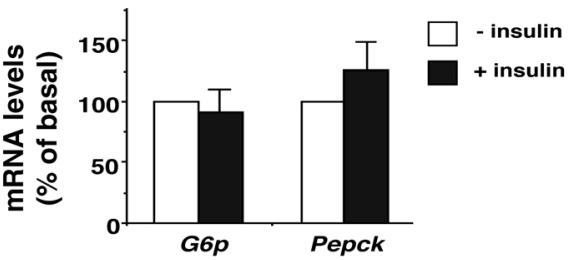

Figure 1

Northern blot analysis of G6p and Pepck expression in LLC. (a) Timecourse analysis of dex/cAMP-induced gene expression. Cell monolayers were incubated with dex/cAMP for the indicated periods of time. Thereafter, mRNA was extracted and size-fractionated on denaturing formaldehyde/agarose gels prior to membrane transfer and Northern hybridization, with the cDNA probes indicated to the left of the autoradiograms. (b) Lack of insulin effect on G6p and Pepck expression. LLC cells were incubated in serum-free medium in the absence (lanes 1 and 4 ) or presence of dex/cAMP for 4 hours (lanes 2 and 3 ) or 16 hours (lanes 5 and 6 ), followed by the addition of insulin (lanes 3 and 6 ) for 6 hours. mRNA isolation and Northern blot analysis were performed as described in Methods. (c) Mean \pm SEM of the percentage of insulin inhibition of dex/cAMP-induced expression at 16 hours was calculated from three independent experiments using densitometric scanning of the autoradiograms. mRNA loading was normalized by subsequent hybridization with a $\beta$-actin probe.

Insulin-dependent Foxo1 and Foxo3 phosphorylation. Cells were incubated in serum-free DMEM supplemented with $0.1 \%$ BSA or dex/cAMP for 16 hours and then stimulated with insulin $(100 \mathrm{nM})$ for the indicated times. At the end of incubation, Foxo1-transfected cells were solubilized and immunoprecipitated with anti-HA mAb 12CA5 (Boehringer Mannheim GmbH), while Foxo3-transfected cells were immunoprecipitated with anti-c-Myc mAb 9E10 (Santa Cruz Biotechnology Inc.). Immune complexes were resolved on $8 \%$ SDS-PAGE and transferred to nitrocellulose filters. The membranes were sequentially reprobed with anti-phospho $\mathrm{T}^{24}, \mathrm{~S}^{253}, \mathrm{~S}^{316}$, and anti-Foxo1 or Foxo3 antisera (Upstate Biotechnology Inc., Lake Placid, New York, USA) to normalize the amount of phosphate incorporated into each band for the amount of protein applied to the gel. Western blot analysis was performed using detection of the immune complexes with horseradish peroxidase-conjugated anti-rabbit IgG (enhanced chemiluminescence [ECL]; Amersham Pharmacia Biotech AB, Piscataway, New Jersey, USA).

Immunofluorescence. LLC cells were transiently transfected with pCMV5/c-Myc encoding Foxo1 or Foxo3 using Lipofectamine (Life Technologies Inc., Rockville, Maryland, USA) according to the manufacturer's protocol $(45,47)$. After 24 hours, cells were seeded into a four-well slide culture chamber (Nalge Nunc Corp., Naperville, Illinois, USA). Cells were incubated in serum-free medium for 16 hours and then stimulated with insulin $(100 \mathrm{nM})$ for the indicated times. Fixation of cells and incubation with anti-c-Myc mAb (9E10) and secondary $\mathrm{Ab}$ have been described previously (47).

\section{Results}

Hormonal regulation of G6p and Pepck mRNAs in LLC cells. Under basal culture conditions, Pepck and G6p mRNAs are expressed at very low levels in LLC cells (Figure 1, a and b, lane 1). Addition of dex/cAMP to the culture medium results in approximately tenfold induction of both mRNAs. The increase in G6p occurs following 8 hours of the addition of dex/cAMP, peaks at 16 hours, and disappears by 24 hours. In contrast, the increase in Pepck is detectable by 2 hours and persists after 24 hours of dex/cAMP treatment (Figure 1a). To study the ability of insulin to affect this response, LLC cells were treated with dex/cAMP for either 4 or 16 hours (Figure1b, lanes 1-3 and 4-6, respectively), and then exposed to insulin for 6 hours. At both time points, the effect of dex/cAMP was not inhibited by insulin (Figure 1, b, lane 3, and 6), indicating that Pepck and G6p are not regulated by insulin in LLC cells. Several experiments are summarized in Figure 1c.

The lack of insulin responsiveness cannot be ascribed to the absence of IRs, which are expressed in LLC cells in sizable amounts (Figure 2, lane 2), as are IGF-1 receptors (lane 4).

Expression of different Foxo isoforms in LLC cells and hepatocytes. Next, we compared expression patterns of Foxo

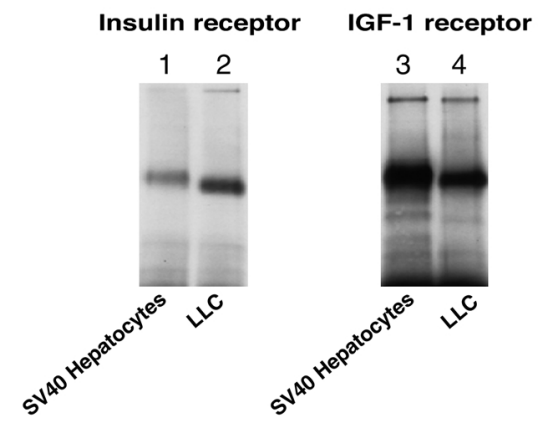

Figure 2

Immunodetection of insulin and IGF-1 receptors in LLC cells. Detergent extracts were prepared from SV40-transformed hepatocytes (lanes 1 and 3 ) and LLC cells (lanes 2 and 4) and immunoprecipitated with antisera against IR (lanes 1 and 2) and IGF-1 receptor (lanes 3 and 4). Equal amounts of protein extracts were used for both experiments. 


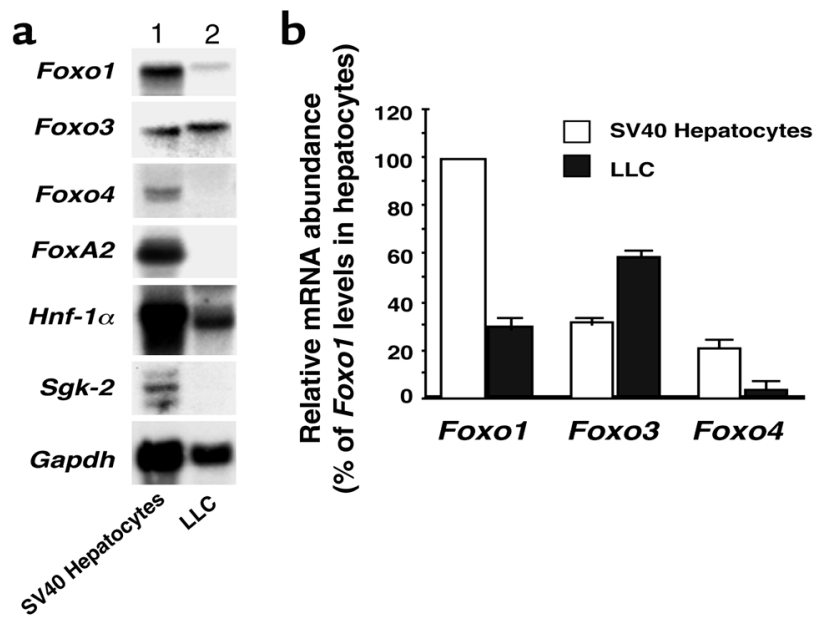

isoforms in LLC cells and SV40 hepatocytes (5). As we have shown previously, Foxo1 is the main Foxo isoform in SV40 (tsA)-transformed hepatocytes (45). Its expression in LLC cells is reduced by approximately $70 \%$ compared with SV40 hepatocytes (Figure 3a, top panel, lanes 1 and 2). Foxo3 is the most abundant Foxo isoform in LLC cells. Its levels are approximately $60 \%$ of those of Foxo1 in SV40 hepatocytes (Figure 3a, second panel from top, lanes 1 and 2). Finally, Foxo4 is expressed at low levels in SV40 hepatocytes, but not in LLC cells (Figure 3, a and b). The forkhead protein Foxa2 (HNF3 $\beta$ ), which has been suggested to participate in regulation of Pepck and G6p expression $(63,64)$,

\section{Figure 3}

Expression of Foxo isoforms in hepatocytes and LLC cells. (a) mRNA was isolated from SV40-transformed hepatocytes and LLC cells as indicated in Methods and hybridized with the probes indicated to the left of the autoradiogram. Exposure time was 12 hours for all probes, except Gapdh, which was exposed for 1 hour. (b) Quantification of the data in a. The signal obtained with each probe was quantitated by scanning densitometry of the autoradiogram. To evaluate the relative expression of the three Foxo isoforms, the densitometric data were corrected for the specific activity of each Foxo probe. The highest level of Foxo expression (Foxo 1 in hepatocytes) was set to $100 \%$. Data were normalized by hybridization of the blots with a Gapdh probe. a

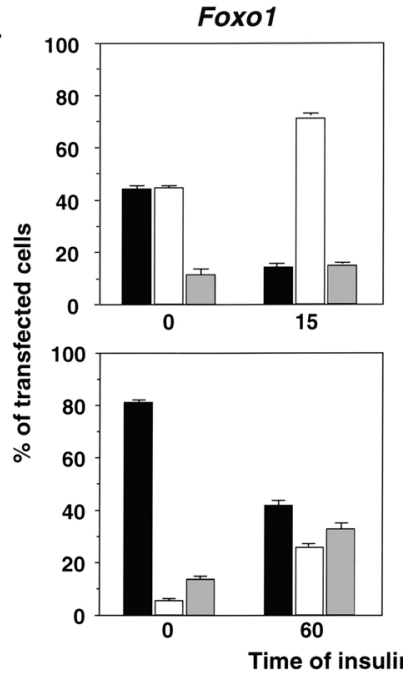

Foxo3
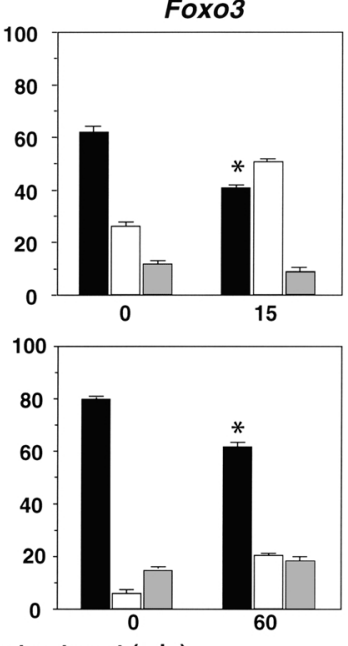

is likewise absent from LLC cells. The transcription factor Hnf- $1 \alpha$ has been implicated in the regulation of Pepck and G6p in the kidney $(22,65-70)$ and is expressed at similar levels in both cell types. Finally, the Foxo kinase Sgk-2 $(41,50)$ is not expressed in LLC cells. These data are consistent with the possibility that lack of insulin inhibition on gluconeogenic gene expression may result from reduced expression of Foxo 1 and/or the Foxo kinase Sgk-2.

Subcellular localization of Foxo1 and Foxo3 in LLC cells. Insulin has been shown to alter Foxo subcellular distribution (47). Thus, we analyzed insulin-induced nuclear export of Foxo 1 and Foxo3 in the presence and

\section{Figure 4}

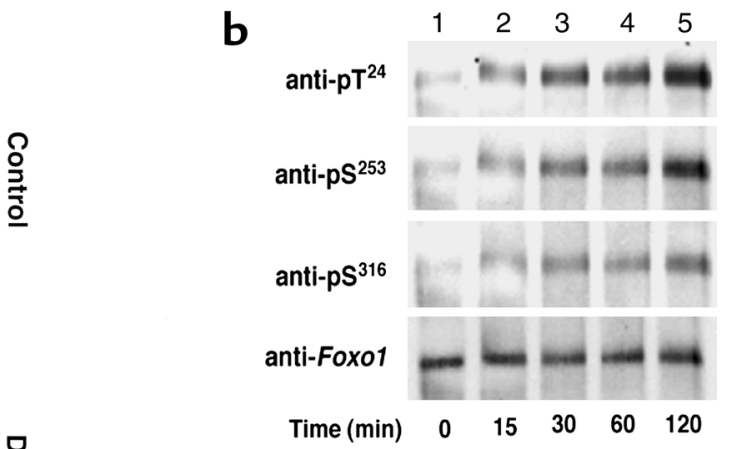

(a) Subcellular localization of Foxo1 and Foxo3 in LLC cells. Cells at approximately $50 \%$ confluence were transiently transfected with c-Myc-tagged Foxo1 or c-Myc-tagged Foxo3. After transfection, cells were seeded into four-well slide culture chambers, cultured overnight in serum-free medium, and incubated in the absence (upper panels) or presence (lower panels) of dex/cAMP for 16 hours. Thereafter, they were treated with insulin $(100 \mathrm{nM})$ for the indicated periods of time. Epitope-tagged Foxo 1 and Foxo3 were visualized with anti-c-Myc mAb and FITC-conjugated anti-mouse IgG. At least 200 transfected cells were visually scored for localization of transfected proteins in each experiment. Data represent mean \pm SEM from three independent transfection experiments. ${ }^{*} P<0.01$ between the number of cells with nuclear staining in Foxo1- and Foxo3-expressing cells by one-factor ANOVA. (b) Insulin-induced Foxo1 phosphorylation. LLC cells were transduced with adenovirus encoding WT Foxo1. After 24 hours, cells were stimulated with insulin (100 nM) for the indicated lengths of time. At the end of the incubation, cells were harvested and detergent extracts were subjected to immunoprecipitation with anti-HA Ab, followed by sequential immunoblotting with anti-phospho-specific Ab's or anti-Foxo1 Ab, as indicated next to each panel. A representative experiment is shown. 
absence of dex/cAMP using immunofluorescence to detect c-Myc-tagged Foxo1 or Foxo3 following transient transfections. Individual cells were scored according to whether they showed exclusively cytoplasmic, exclusively nuclear, or diffuse immunostaining. In the absence of dex/cAMP, insulin treatment for 15 minutes decreased the percentage of cells showing exclusively nuclear Foxo1 staining from 45 to $12 \%$, while the percentage of Foxo3-positive cells with nuclear staining decreased from 62 to $42 \%$ (Figure 4a, upper panels). In the presence of dex/cAMP, insulin-induced translocation was considerably delayed. Thus, results at 1 hour of treatment are shown. Nuclear staining decreased from 80 to $45 \%$ of Foxo1-expressing cells and from 80 to $60 \%$ of Foxo3-expressing cells (Figure 4, lower panels). The difference in nuclear localization between Foxo3- and Foxo1-expressing cells was statistically significant $(P<0.01$ by ANOVA) under all treatment conditions, except in the absence of insulin in dex/cAMP-treated cells (lower panels). These data show that insulin increases nuclear export of Foxo in kidney cells, while dex/cAMP antagonizes insulin's effect. From these data we cannot conclude whether dex/cAMP affects insulin-induced Foxo1 translocation directly or indirectly. However, it is possible to suggest that Foxo 1 is more efficiently translocated from the nucleus to the cytoplasm than Foxo3. The latter findings are consistent with the suggestion that these two proteins are differentially regulated by insulin (46).

Analysis of insulin-induced phosphorylation by way of anti-phosphopeptide Ab's revealed that Foxo1 was phosphorylated on three Akt consensus sites $-\mathrm{T}^{24}$, $\mathrm{S}^{253}$, and $\mathrm{S}^{316}$ - in an insulin-dependent manner (Figure $4 \mathrm{~b}$ ), whereas Foxo3 was phosphorylated on two dif- ferent sites, $\mathrm{T}^{24}$ and $\mathrm{S}^{253}$ (data not shown). We could not assess the phosphorylation of Foxo3 $\mathrm{S}^{316}$ for lack of a suitable phospho-specific $A b$.

Foxo1 confers insulin-responsive G6p expression in LLC cells. Next, we asked whether expression of Foxo1 would confer insulin regulation onto G6p and Pepck expression. In cells transduced with Foxo1, both Pepck and G6p were still detectable after 24 hours of dex/cAMP treatment, suggesting that Foxo1 extends the duration of this effect (Figure 5a, lanes 1-3). Foxo1 did not increase basal G6p and Pepck levels, but resulted in approximately $60 \%$ increase of dex/cAMP-induced G6p expression $(P<0.05)$ and approximately $25 \%$ increase of Pepck expression ( $P=$ NS) (Figure 5b, lane 2). Addition of insulin blunted the effect of dex/cAMP on G6p in a time-dependent manner, reaching the maximal effect after 6 hours of exposure to the hormone (Figure 5b, compare lanes 2-5 with 6-9). No significant effect was seen on Pepck (5b, middle panel). The effect of insulin was studied at two different time points of dex/cAMP treatment. After either 4 or 16 hours of dex/cAMP exposure, in Foxo1-expressing cells insulin inhibited the dex/cAMP-induced increment in G6p expression by $60 \%(P<0.05$ by ANOVA), while no inhibition was observed for Pepck. Data from several experiments are summarized in Figure $5 c$.

Effects of dominant negative and constitutively active Foxo1 mutants on G6p and Pepck expression. To determine whether Foxo1 induces gene expression through a dex/cAMP-dependent mechanism, we analyzed the ability of gain- and loss-of-function Foxo1 mutants to affect dex/cAMP-induced gene expression. It has been shown that a truncated Foxo1 mutant lacking the transactivation domain $(\Delta 256)$ acts as a dominant neg-

\section{Figure 5}

Foxo1 expression confers insulin inhibition on G6p, but not on Pepck. (a) Time-course analysis of dex/cAMP effect in cells transduced with Foxo1. LLC cells were transduced with adenovirus encoding WT Foxo1 and incubated with dex/cAMP for the indicated periods of time. At the end of the incubation, mRNA was extracted and analyzed by Northern blot analysis using the relevant cDNA probes. (b) Time-course analysis of insulin effect. LLC cells were transduced with Foxo 1 adenovirus. After 48 hours, the medium was replaced with serum-free medium, and incubation was continued overnight. Thereafter, dex/cAMP was added for 16 hours, followed by 6 hours of treatment in the presence (lanes 2-5) or in the absence of insulin (lanes 6-9) for various lengths of time, as indicated. Northern blot analysis was performed as described above. (c) Northern blot of G6p and Pepck mRNAs following transduction of LLC cells with adenoviral vector encoding Foxo1. LLC cells were incubated in serum-free medium overnight before addition of dex/cAMP for 4 hours (lanes 2 and 3 ) or 16 hours (lanes 5 and 6), followed by insulin stimulation for 6 hours (lanes 3

and 6). Thereafter, mRNA was isolated and Northern blot analysis was performed with cDNA probes encoding G6p (upper panel), Pepck (middle panel), and $\beta$-actin (lower panel). A representative experiment is shown, and data from three independent adenoviral transductions are summarized in (d). Mean \pm SEM of the percentage of insulin inhibition of dex/cAMP-induced expression at 16 hours was calculated from three independent experiments using densitometric scanning of the autoradiograms. The mRNA loading was normalized by subsequent hybridization with a $\beta$-actin probe. ${ }^{*} P<0.05$ ANOVA. a
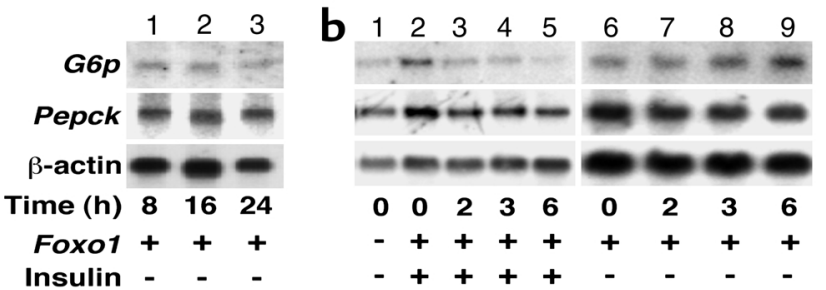

C
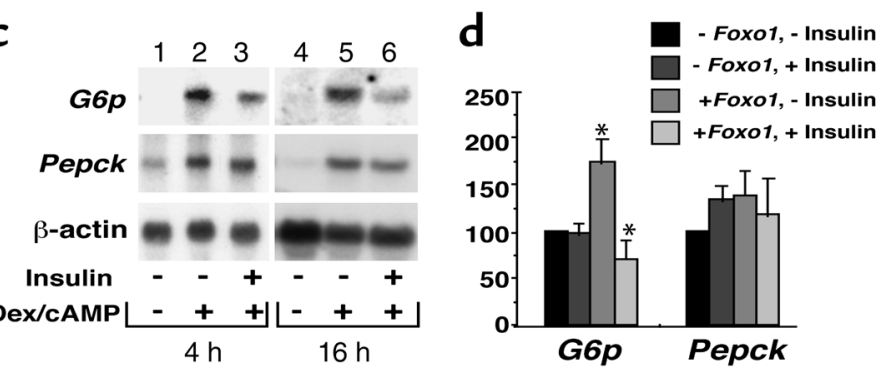


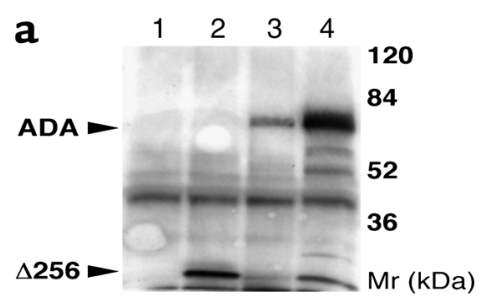

b

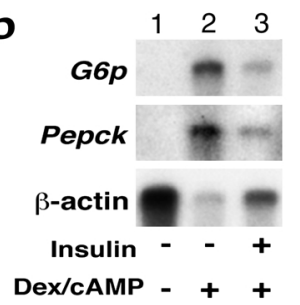

C

Kidney

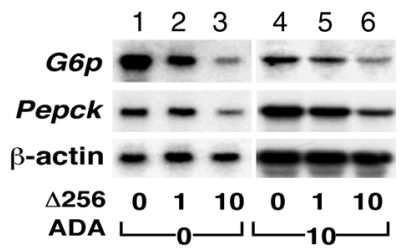

d

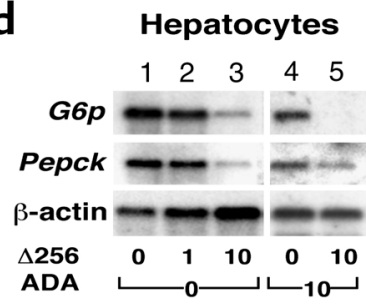

\section{Figure 6}

Effects of constitutively active and dominant negative Foxo 1 mutants on G6p and Pepck in LLC cells and in primary hepatocytes. (a) Expression of HA-tagged $\Delta 256$ - and ADA-Foxo1 mutants in LLC cells was measured as indicated in Methods. Lane 1, untransduced LLC cells; lane 2, LLC cells transduced with the $\Delta 256$ mutant; lane 3, LLC cells transduced with the ADA mutant; lane 4, LLC cells cotransduced with both $\Delta 256$ and ADA mutants at a 1:10 moi. (b) Insulin inhibits G6p and Pepck in primary cultures of mouse hepatocytes. Hepatocytes were isolated as described in Methods. Cell monolayers were incubated in serum-free medium for 4 hours before the addition of dex/cAMP for 8 hours. Thereafter, cells were incubated in the absence (lanes 1 and 2) or in the presence of insulin (lane 3 ) for 6 hours. The data are representative of three separate hepatocyte preparations. (c) Effects of Foxo1 mutants on dex/cAMP-induced G6p and Pepck expression in LLC cells. Cells were transduced with the $\Delta 256$ mutant at increasing moi, in the absence (lanes 1-3) or presence (lanes 4-6) of a fixed amount of ADA-Foxo1 mutant. After overnight incubation in serum-free

medium, cells were incubated in the presence of dex/cAMP for 8 hours. A representative experiment is shown. (d) Effects of Foxo1 mutants on dex/cAMP-induced G6p and Pepck expression in primary cultures of mouse hepatocytes. After transduction with $\Delta 256-$ and/or ADA-Foxo1 mutant adenoviruses, hepatocyte cultures were incubated in serum-free medium for 4 hours before the addition of dex/cAMP for 8 hours. A representative experiment is shown, and data from three separate experiments for each cell type are summarized in Figure 7 .

ative inhibitor of Foxo1-mediated transcription of an Igfbp-1 reporter construct containing an insulin response element (IRE) (refs. 40, 71 and our unpublished observation). In contrast, a phosphorylationdefective mutant, in which all three potential Akt phosphorylation sites have been replaced by nonphosphorylatable amino acids (ADA), cannot be excluded from the nucleus in response to insulin and is constitutively active $(39,40,47,51,71)$. Transduction of LLC cells with adenoviral vectors encoding $\Delta 256$-Foxo 1 and/or ADA-Foxo1 resulted in readily detectable expression of the two mutant proteins (Figure 6a, lanes $2-4)$. In these loss-of-function experiments, we also studied the effect of Foxo1 in primary cultures of mouse hepatocytes. As shown in Figure 6b, addition of dex/cAMP resulted in a brisk increase of both G6p and Pepck expression, which was readily inhibited by insulin treatment (lanes 2 and 3). Expression of the $\Delta 256$ mutant resulted in a dose-dependent decrease of dex/cAMP-induced G6p expression up to approximately $80 \%$ in LLC cells and $90 \%$ in primary hepatocytes (Figure 6, c and d, lanes 1-3, and Figure 7, a and

\footnotetext{
Figure 7

Summary of the effects of the constitutively active and dominant negative Foxo1 mutants on dex/cAMP-induced gene expression. Data from three independent experiments in LLC cells and three different hepatocyte preparations are expressed as mean \pm SEM of the percentage of inhibition of dex/cAMP-induced G6p ( $\mathbf{a}$ and $\mathbf{c}$ ) and Pepck (b and $\mathbf{d}$ ) expression by the dominant negative $\Delta 256$ Foxo 1 mutant in the absence (filled bars) or presence (open bars) of the constitutively active ADA Foxo1 mutant. ( $\mathbf{a}$ and $\mathbf{b}$ ) Summary of the results in LLC cells. (c and d) Summary of the results in primary hepatocyte cultures. ${ }^{*} P<0.01$ by ANOVA.
}

c). Likewise, Pepck expression decreased up to $70 \%$ in LLC cells and $80 \%$ in primary hepatocytes (Figures 6, c and d, lanes 1-3, and Figure 7, b and d) $(P<0.001)$.

Similar results were obtained in cells cotransduced with the constitutively active mutant at a fixed moi and increasing concentrations of dominant negative mutant (Figure 6, c and d, lanes 4-6, and Figure 7, a-d). These data indicate that a dominant negative Foxo1 protein can affect the dex/cAMP-induced rise of G6p and Pepck and effectively prevent the increase due to a constitutively active Foxo1 mutant.

\section{Discussion}

In this study we demonstrate that cultured kidney epithelial cells lack insulin inhibition of Pepck and

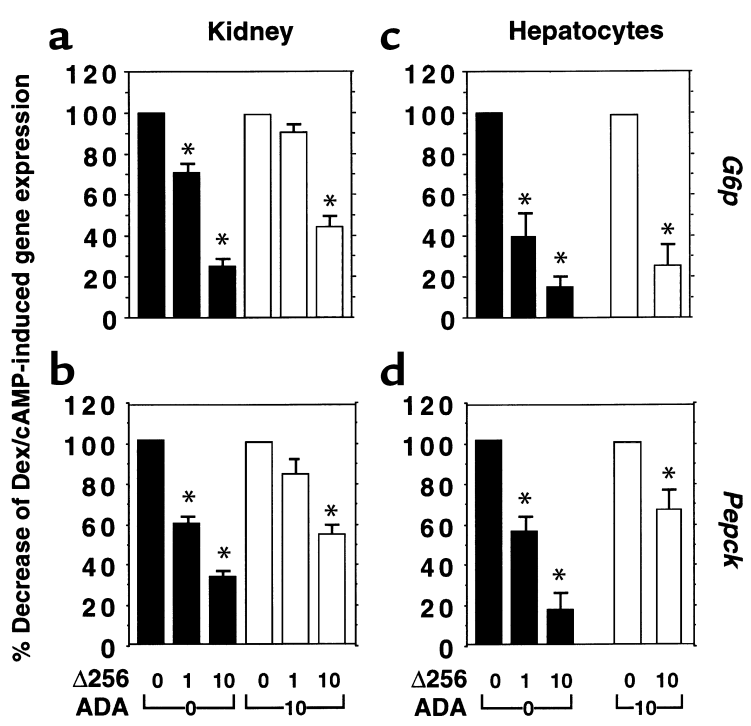


G6p expression. We seized upon this observation to investigate the role of the forkhead transcription factor Foxo1 in this process. We show that insulin's failure to inhibit Pepck and G6p in LLC cells correlates with low Foxo1 mRNA levels and that transduction with a Foxo 1 adenovirus confers insulin inhibition on dex/cAMP-induced G6p, but not Pepck expression. These data support the hypothesis that Foxo1 regulates expression of the endogenous G6p in an insulindependent manner. We also show that a dominant negative Foxo1 mutant effectively prevents the dex/cAMP-induced increases in G6p and Pepck expression in both LLC cells and primary hepatocytes, consistent with the possibility that Foxo1 functions as a bona fide insulin-regulated transcription factor on endogenous genes. It bears emphasizing that this point had, thus far, eluded demonstration.

Foxo isoforms in LLC cells. The main Foxo isoform in LLC cells is Foxo3, which shares common structural features with Foxo1 (38). We show that Foxo3 is less responsive than Foxo1 to insulin-induced nuclear translocation. This difference is consistent with the observation that insulin inhibits Foxo3-dependent gene expression in hepatoma cells less markedly than Foxo1-dependent gene expression (46). A potential explanation for the lackluster insulin response of Foxo3 is that LLC cells lack one of the Foxo kinases, Sgk-2 $(41,50)$. We suggest that Foxo1 is the principal insulin-responsive Foxo isoform, consistent with its distribution in insulin-responsive tissues (unpublished observation). Moreover, loss- and gain-of-function Foxo1 mutations modulate insulin sensitivity in mice (72). We do not know whether differences in nuclear export rates between Foxo 1 and Foxo 3 can account for the different properties of these two proteins to mediate insulin inhibition of gene expression. From our limited analysis, we cannot conclude that nuclear exclusion is the sole or even the main mechanism by which insulin regulates Foxo1 (see below).

Specificity of Foxo1 regulation of gene expression. The ability of Foxo1 to suppress dex/cAMP-induced G6p, but not Pepck, expression in an insulin-dependent fashion suggests that different mechanisms regulate insulin inhibition of these two target genes. Since our studies analyzed expression of the endogenous genes, we are not in a position to comment on the cis-acting elements required for the Foxo1 effect. However, the magnitude of the Foxo1 effect on G6p inhibition by insulin is consistent with that observed in cultured liver cells or in animal models (30).

In the human and murine G6p promoters, two elements are required for transcriptional repression by insulin: A (nt -231 to -199 ) and B (nt -198 to -159$)$. Region A binds the accessory factor hepatic nuclear factor- $1 \alpha$, which is expressed at high levels in LLC cells (refs. 70, 73 and this study). Region B contains three copies of a consensus IRS. This region has been shown to bind a GST-Foxo1 fusion protein (74). When used in a reporter gene assay in hepatoma cells, the same ele- ment bestowed insulin sensitivity and Foxo1 dependence, consistent with a role in Foxo1-mediated gene expression (57). Our data complement this body of observations and suggest that G6p is a physiologic target of Foxo1-mediated insulin inhibition.

Mechanisms of transcriptional regulation. The ability of Foxo1 to modulate G6p expression in a dex/cAMP-dependent manner is consistent with a model in which Foxo1 requires glucocorticoids and cAMP to regulate G6p transcription. This conclusion is supported by a variety of observations presented in this study, including the lack of effect of Foxo1 on basal G6p expression, the additive effect of Foxo 1 and dex/cAMP to increase G6p mRNA, and the failure of the dominant negative Foxo1 to decrease G6p below the levels observed in the absence of dex/cAMP. This observation is similar to the reported requirement of HNF-1 in cAMP stimulation of G6p in LLC cells (68). Thus, Foxo 1 appears to act as an accessory factor of the glucocorticoid/cAMP response that is inhibited by insulin, in part through nuclear exclusion.

The mechanism of Foxo 1 action on Pepck appears to be quite different. In this case, Foxo1 is unable to confer insulin sensitivity on kidney Pepck expression, but the dominant negative Foxo1 can inhibit dex/cAMP-induced Pepck expression in both kidney and hepatocytes. These data are consistent with the possibility that Foxo1 regulates Pepck indirectly, as already suggested by Hall and coworkers based on reporter gene studies in hepatoma cells (56). The mechanism by which the dominant negative affects the glucocorticoid response is at present unclear. Based on the work of Nasrin and colleagues, one possible explanation is that the truncated Foxo1 prevents the recruitment of steroid receptor coactivator SRC to the glucocorticoid response unit (46). Interestingly, a different forkhead mutant, encoding a truncated FOXA2, has a similar inhibitory effect in hepatoma cells (63). The recent demonstration that Foxo1 can function both as a coactivator or a corepressor of transcription by interacting with nuclear hormone receptors provides an additional mechanism to explain the effect of the dominant negative Foxo1 on Pepck (75).

Conclusions. The kidney contributes a small, but significant, fraction to overall glucose release in humans and rodents. The exact magnitude of this contribution remains controversial, probably as a result of methodological differences (76). However, there is agreement that renal glucose production, like hepatic glucose production, is increased in diabetes (77-79). The ability of a dominant negative Foxo1 to partially inhibit G6p and Pepck expression in the kidney could be exploited in a gene therapy approach to reduce glucose production in diabetic patients without incurring the potentially lethal complication of hypoglycemia.

\section{Acknowledgments}

Supported by NIH grant DK-57539 and by a grant from the American Diabetes Association to D. Accili. We 
thank Norman P. Curthoys (Colorado State University) for his generous gift of LLC-PK1-FBPase ${ }^{+}$cells and the following colleagues for providing cDNA probes: Janice Y. Chou (NIH), Richard W. Hanson (Case Western Reserve University), Markus Stoffel (Rockefeller University), Masato Kasuga and Wataru Ogawa (Kobe University), Karen Arden and William Biggs, III (University of California, San Diego). We thank Alan Tall (Columbia University) for making his laboratory available for the isolation of primary hepatocytes cultures.

1. Saltiel, A.R. 2001. New perspectives into the molecular pathogenesis and treatment of type 2 diabetes. Cell. 104:517-529.

2. Cherrington, A.D. 1999. Banting Lecture 1997. Control of glucose uptake and release by the liver in vivo. Diabetes. 48:1198-1214.

3. Lauro, D., et al. 1998. Impaired glucose tolerance in mice with a targeted impairment of insulin action in muscle and adipose tissue. Nat. Genet. 20:294-298.

4. Michael, M.D., et al. 2000. Loss of insulin signaling in hepatocytes leads to severe insulin resistance and progressive hepatic dysfunction. Mol. Cell. 6:87-97.

5. Rother, K.I., et al. 1998. Evidence that IRS-2 phosphorylation is required for insulin action in hepatocytes. J. Biol. Chem. 273:17491-17497.

6. Di Cola, G., Cool, M.H., and Accili, D. 1997. Hypoglycemic effect of insulin-like growth factor-1 in mice lacking insulin receptors. J. Clin. Invest. 99:2538-2544.

7. Gerich, J.E., Meyer, C., Woerle, H.J., and Stumvoll, M. 2001. Renal gluconeogenesis: its importance in human glucose homeostasis. Diabetes Care. 24:382-391.

8. Stumvoll, M., et al. 1995. Uptake and release of glucose by the human kidney. Postabsorptive rates and responses to epinephrine. J. Clin. Invest. 96:2528-2533

9. Stumvoll, M., Meyer, C., Mitrakou, A., Nadkarni, V., and Gerich, J.E. 1997. Renal glucose production and utilization: new aspects in humans. Diabetologia. 40:749-757.

10. Cersosimo, E., Judd, R.L., and Miles, J.M. 1994. Insulin regulation of renal glucose metabolism in conscious dogs. J. Clin. Invest. 93:2584-2589.

11. Owen, O.E., Felig, P., Morgan, A.P., Wahren, J., and Cahill, G.F. 1969. Liver and kidney metabolism during prolonged starvation. J. Clin. Invest. 48:574-583.

12. Felig, P., Marliss, E., Owen, O.E., and Cahill, G.F. 1969. Blood glucose and gluconeogenesis in fasting man. Arch. Intern. Med. 123:293-298.

13. Meyer, C., Dostou, J.M., and Gerich, J.E. 1999. Role of the human kidney in glucose counterregulation. Diabetes. 48:943-948.

14. Meyer, C., Nadkarni, V., Stumvoll, M., and Gerich, J. 1997. Human kidney free fatty acid and glucose uptake: evidence for a renal glucose-fatty acid cycle. Am. J. Physiol. 273:E650-654.

15. Ekberg, K., et al. 1999. Contributions by kidney and liver to glucose production in the postabsorptive state and after $60 \mathrm{~h}$ of fasting. Diabetes. 48:292-298.

16. Cersosimo, E., Ajmal, M., Naukam, R.J., Molina, P.E., and Abumrad, N.N 1997. Role of the kidney in plasma glucose regulation during hyperglycemia. Am. J. Physiol. 272:E756-761.

17. Cersosimo, E., Garlick, P., and Ferretti, J. 1999. Insulin regulation of renal glucose metabolism in humans. Am. J. Physiol. 276:E78-84.

18. Wirthensohn, G., and Guder, W.G. 1986. Renal substrate metabolism. Physiol. Rev. 66:469-497.

19. Goligorsky, M.S., Osborne, D., Howard, T., Hruska, K.A., and Karl, I.E. 1987. Hormonal regulation of gluconeogenesis in cultured proximal tubular cells: role of cytosolic calcium. Am. J. Physiol. 253:F802-809.

20. Watford, M., and Mapes, R.E. 1990. Hormonal and acid-base regulation of phosphoenolpyruvate carboxykinase mRNA levels in rat kidney. Arch. Biochem. Biophys. 282:399-403.

21. Hwang, J.J., and Curthoys, N.P. 1991. Effect of acute alterations in acidbase balance on rat renal glutaminase and phosphoenolpyruvate carboxykinase gene expression. J. Biol. Chem. 266:9392-9396.

22. Kaiser, S., and Curthoys, N.P. 1991. Effect of $\mathrm{pH}$ and bicarbonate on phosphoenolpyruvate carboxykinase and glutaminase mRNA levels in cultured renal epithelial cells. J. Biol. Chem. 266:9397-9402.

23. Gstraunthaler, G., et al. 2000. Differential expression and acid-base regulation of glutaminase mRNAs in gluconeogenic LLC-PK(1)-FBPase $(+)$ cells. American Journal of Physiology - Renal Physiology. 278:F227-237.

24. Liu, W., et al. 1998. PMA and staurosporine affect expression of the PCK gene in LLC-PK1-F+ cells. Am. J. Physiol. 275:F361-369.

25. Holcomb, T., Curthoys, N.P., and Gstraunthaler, G. 1995. Subcellular localization of PEPCK and metabolism of gluconeogenic substrains of renal cell lines. Am. J. Physiol. 268:C449-C457.

26. Iynedjian, P.B., Ballard, F.J., and Hanson, R.W. 1975. The regulation of phosphoenolpyruvate carboxykinase (GTP) synthesis in rat kidney cortex. The role of acid-base balance and glucocorticoids. J. Biol. Chem 250:5596-5603.

27. Parry, D.M., and Brosnan, J.T. 1978. Glutamine metabolism in the kidney during induction of, and recovery from, metabolic acidosis in the rat. Biochem. J. 174:387-396.

28. Burch, H.B., et al. 1978. Distribution along the rat nephron of three enzymes of gluconeogenesis in acidosis and starvation. Am. J. Physiol. 235:F246-253.

29. Pollock, A.S., and Long, J.A. 1989. The $5^{\prime}$ region of the rat phosphoenolpyruvate carboxykinase gene confers $\mathrm{pH}$ sensitivity to chimeric genes expressed in renal and liver cell lines capable of expressing PEPCK Biochem. Biophys. Res. Commun. 164:81-87.

30. O’Brien, R.M., and Granner, D.K. 1996. Regulation of gene expression by insulin. Physiol. Rev. 76:1109-1161.

31. Hanson, R.W., and Reshef, L. 1997. Regulation of phosphoenolpyruvate carboxykinase (GTP) gene expression. Annu. Rev. Biochem. 66:581-611.

32. Sutherland, C., O’Brien, R.M., and Granner, D.K. 1995. Phosphatidylinositol 3-kinase, but not $\mathrm{p} 70 / \mathrm{p} 85$ ribosomal $\mathrm{S} 6$ protein kinase, is required for the regulation of phosphoenolpyruvate carboxykinase (PEPCK) gene expression by insulin. Dissociation of signaling pathways for insulin and phorbol ester regulation of PEPCK gene expression. $J$. Biol. Chem. 270:15501-15506.

33. Cichy, S.B., et al. 1998. Protein kinase B/Akt mediates effects of insulin on hepatic insulin-like growth factor-binding protein-1 gene expression through a conserved insulin response sequence. J. Biol. Chem. 273:6482-6487.

34. Kotani, K., et al. 1999. Dominant negative forms of Akt (protein kinase B) and atypical protein kinase $\mathrm{C} \lambda$ do not prevent insulin inhibition of phosphoenolpyruvate carboxykinase gene transcription. J. Biol. Chem. 274:21305-21312.

35. Dickens, M., Svitek, C.A., Culbert, A.A., O’Brien, R.M., and Tavare, J.M. 1998. Central role for phosphatidylinositide 3-kinase in the repression of glucose-6-phosphatase gene transcription by insulin. J. Biol. Chem. 273:20144-20149.

36. Liao, J., Barthel, A., Nakatani, K., and Roth, R.A. 1998. Activation of protein kinase B/Akt is sufficient to repress the glucocorticoid and cAMP induction of phosphoenolpyruvate carboxykinase gene. J. Biol. Chem. 273:27320-27324.

37. Agati, J.M., Yeagley, D., and Quinn, P.G. 1998. Assessment of the roles of mitogen-activated protein kinase, phosphatidylinositol 3-kinase, protein kinase $B$, and protein kinase $C$ in insulin inhibition of CAMPinduced phosphoenolpyruvate carboxykinase gene transcription. J. Biol. Chem. 273:18751-18759.

38. Kaestner, K.H., Knochel, W., and Martinez, D.E. 2000. Unified nomenclature for the winged helix/forkhead transcription factors. Genes Dev. 14:142-146.

39. Brunet, A., et al. 1999. Akt promotes cell survival by phosphorylating and inhibiting a Forkhead transcription factor. Cell. 96:857-868.

40. Biggs, W.H.R., Meisenhelder, J., Hunter, T., Cavenee, W.K., and Arden, K.C. 1999. Protein kinase B/Akt-mediated phosphorylation promotes nuclear exclusion of the winged helix transcription factor FKHR1. Proc. Natl. Acad. Sci. USA. 96:7421-7426.

41. Brunet, A., et al. 2001. Protein kinase SGK mediates survival signals by phosphorylating the forkhead transcription factor FKHRL1 (FOXO3a). Mol. Cell Biol. 21:952-965.

42. Cahill, C.M., et al. 2000. Phosphatidylinositol PI-3 kinase signaling inhibits DAF-16 DNA binding and function via 14-3-3 dependent and 14-3-3 independent pathways. J. Biol. Chem. 276:13402-13410.

43. del Peso, L., Gonzalez, V.M., Hernandez, R., Barr, F.G., and Nunez, G. 1999. Regulation of the forkhead transcription factor FKHR, but not the PAX3-FKHR fusion protein, by the serine/threonine kinase Akt. Onco gene. 18:7328-7333.

44. Durham, S.K., et al. 1999. FKHR binds the insulin response element in the insulin-like growth factor binding protein-1 promoter. Endocrinology. 140:3140-3146.

45. Nakae, J., Park, B.-C., and Accili, D. 1999. Insulin stimulates phosphorylation of the forkhead transcription factor FKHR on serine 253 through a Wortmannin-sensitive pathway. J. Biol. Chem. 274:15982-15985.

46. Nasrin, N., et al. 2000. DAF-16 recruits the CREB-binding protein coactivator complex to the insulin-like growth factor binding protein 1 promoter in HepG2 cells. Proc. Natl. Acad. Sci. USA. 97:10412-10417.

47. Nakae, J., Barr, V., and Accili, D. 2000. Differential regulation of gene expression by insulin and IGF-1 receptors correlates with phosphorylation of a single amino acid residue in the forkhead transcription factor FKHR. EMBO J. 19:989-996.

48. Rena, G., Guo, S., Cichy, S.C., Unterman, T.G., and Cohen, P. 1999. Phosphorylation of the transcription factor forkhead family member FKHR by protein kinase B. J. Biol. Chem. 274:17179-17183.

49. Tang, E.D., Nunez, G., Barr, F.G., and Guan, K.L. 1999. Negative regulation of the forkhead transcription factor FKHR by Akt. J. Biol. Chem. 274:16741-16746. 
50. Liu, D., Yang, X., and Songyang, Z. 2000. Identification of CISK, a new member of the SGK kinase family that promotes IL-3-dependent survival. Curr. Biol. 10:1233-1236.

51. Guo, S., et al. 1999. Phosphorylation of serine 256 by protein kinase B disrupts transactivation by FKHR and mediates effects of insulin on insulin-like growth factor-binding protein-1 promoter activity through a conserved insulin response sequence. J. Biol. Chem. 274:17184-17192.

52. Datta, S.R., Brunet, A., and Greenberg, M.E. 1999. Cellular survival: play in three Akts. Genes Dev. 13:2905-2927.

53. Medema, R.H., Kops, G.J., Bos, J.L., and Burgering, B.M. 2000. AFX-like forkhead transcription factors mediate cell-cycle regulation by Ras and PKB through p27kip1. Nature. 404:782-787.

54. Dijkers, P.F., et al. 2000. Forkhead transcription factor FKHR-L1 modulates cytokine-dependent transcriptional regulation of p27(KIP1) Mol. Cell Biol. 20:9138-9148.

55. Dijkers, P.F., Medemadagger, R.H., Lammers, J.J., Koenderman, L., and Coffer, P.J. 2000. Expression of the pro-apoptotic bcl-2 family member Bim is regulated by the forkhead transcription factor FKHR-L. Curr. Biol. 10:1201-1204.

56. Hall, R.K., Yamasaki, T., Kucera, T., O’Brien R.M., and Granner, D.K. 2000. Regulation of phosphoenolpyruvate carboxykinase and insulinlike growth factor binding protein-1 gene expression by insulin. J. Biol. Chem. 275:30169-30175.

57. Schmoll, D., et al. 2000. Regulation of glucose-6-phosphatase gene expression by protein kinase Balpha and the forkhead transcription factor FKHR. J. Biol. Chem. 275:36324-36333.

58. Gstraunthaler, G., Pfaller, W., and Kotanko, P. 1985. Lack of fructose1,6-bisphosphatase activity in LLC-PK1 cells. Am. J. Physiol. 248:C181-183

59. Gstraunthaler, G., Gersdorf, E., Fischer, W.M., Joannidis, M., and Pfaller, W. 1990. Morphological and biochemical changes of LLC-PK1 cells during adaptation to glucose-free culture conditions. Ren. Physiol. Biochem. 13:137-153.

60. Honkakoski, P., and Negishi, M. 1998. Protein serine/threonine phosphatase inhibitors suppress phenobarbital-induced Cyp2b10 gene transcription in mouse primary hepatocytes. Biochem. J. 330:889-895.

61. Silver, D.L., Wang, N., and Tall, A.R. 2000. Defective HDL particle uptake in $o b / o b$ hepatocytes causes decreased recycling, degradation, and selective lipid uptake. J. Clin. Invest. 105:151-159.

62. Kitamura, T., et al. 1998. Requirement for activation of the serine-threonine kinase Akt (protein kinase B) in insulin stimulation of protein synthesis but not of glucose transport. Mol. Cell. Biol. 18:3708-3717.

63. Wang, J.C., Stafford, J.M., Scott, D.K., Sutherland, C., and Granner, D.K. 2000. The molecular physiology of hepatic nuclear factor 3 in the regulation of gluconeogenesis. J. Biol. Chem. 275:14717-14721.

64. O’Brien, R.M., et al. 1995. Hepatic nuclear factor 3- and hormone-regulated expression of the phosphoenolpyruvate carboxykinase and insulin- like growth factor-binding protein 1 genes. Mol. Cell Biol. 15:1747-1758. 65. Eisenberger, C.L., Nechushtan, H., Cohen, H., Shani, M., and Reshef, 1992. Differential regulation of the rat phosphoenolpyruvate carboxykinase gene expression in several tissues of transgenic mice. Mol. Cell Biol. 12:1396-1403.

66. Patel, Y.M., Yun, J.S., Liu, J., McGrane, M.M., and Hanson, R.W. 1994. An analysis of regulatory elements in the phosphoenolpyruvate carboxykinase (GTP) gene which are responsible for its tissue-specific expression and metabolic control in transgenic mice. J. Biol. Chem. 269:5619-5628.

67. Holcomb, T., Liu, W., Snyder, R., Shapiro, R., and Curthoys, N.P. 1996. Promoter elements that mediate the $\mathrm{pH}$ response of PCK mRNA in LLCPK1- F+ cells. Am. J. Physiol. 271:F340-346.

68. Cassuto, H., et al. 1997. Involvement of HNF-1 in the regulation of phosphoenolpyruvate carboxykinase gene expression in the kidney. FEBS Lett. 412:597-602.

69. Cissell, M.A., and Chalkley, R. 1999. Characterization of a kidney-specific pattern of chromatin structure in the rat phosphoenolpyruvate carboxykinase gene. Biochim. Biophys. Acta. 1445:299-313.

70. Streeper, R.S., Svitek, C.A., Goldman, J.K., and O'Brien, R.M. 2000. Differential role of hepatocyte nuclear factor- 1 in the regulation of glucose6-phosphatase catalytic subunit gene transcription by cAMP in liver- and kidney-derived cell lines. J. Biol. Chem. 275:12108-12118.

71. Kops, G.J., et al. 1999. Direct control of the Forkhead transcription factor AFX by protein kinase B. Nature. 398:630-634.

72. Nakae, J., Kitamura, T., Biggs, W., Arden, K., and Accili, D. 2001. Mutations of the Forkhead transcription factor FKHR modulate insulin sensitivity in mice. Diabetes. 50 (Suppl. 1):A58 (Abstr 233).

73. Lin, B., Morris, D.W., and Chou, J.Y. 1997. The role of HNF1alpha, HNF3gamma, and cyclic AMP in glucose-6-phosphatase gene activation. Biochemistry. 36:14096-14106.

74. Ayala, J.E., et al. 1999. Conservation of an insulin response unit between mouse and human glucose-6-phosphatase catalytic subunit gene promoters: transcription factor FKHR binds the insulin response sequence. Diabetes. 48:1885-1889.

75. Zhao, H.H., et al. 2001. FKHR functions as a bifunctional nuclear receptor interacting protein with both coactivator and corepressor functions. J. Biol. Chem. 276:27907-27912.

76. Gerich, J.E. 2001. Measurements of renal glucose release. Diabetes. 50:905

77. Mithieux, G., et al. 1996. Glucose-6-phosphatase mRNA and activity are increased to the same extent in kidney and liver of diabetic rats. Diabetes. 45:891-896.

78. Lemieux, G., Aranda, M.R., Fournel, P., and Lemieux, C. 1984. Renal enzymes during experimental diabetes mellitus in the rat. Role of insulin, carbohydrate metabolism, and ketoacidosis. Can. J. Physiol. Pharmacol. 62:70-75.

79. Meyer, C., et al. 1998. Abnormal renal and hepatic glucose metabolism in type 2 diabetes mellitus. J. Clin. Invest. 102:619-624. 